\title{
Adiabatic Creation of Atomic Squeezing in Dark States vs. Decoherences
}

\author{
Z. R. Gong), ${ }^{1}$ Xiaoguang Wang, ${ }^{2}$ and C. P. Sun ${ }^{1}$ \\ ${ }^{1}$ Institute of Theoretical Physics, Chinese Academy of Sciences, Beijing 100190, China \\ ${ }^{2}$ Zhejiang Institute of Modern Physics, Department of Physics, Zhejiang University, Hangzhou 31002\%, China
}

\begin{abstract}
We study the multipartite correlations of the multi-atom dark states, which are characterized by the atomic squeezing beyond the pairwise entanglement. It is shown that, in the photon storage process with atomic ensemble via electromagnetically induced transparency (EIT) mechanism, the atomic squeezing and the pairwise entanglement can be created by adiabatically manipulating the Rabi frequency of the classical light field on the atomic ensemble. We also consider the sudden death for the atomic squeezing and the pairwise entanglement under various decoherence channels. An optimal time for generating the greatest atomic squeezing and pairwise entanglement is obtained by studying in details the competition between the adiabatic creation of quantum correlation in the atomic ensemble and the decoherence that we describe with three typical decoherence channels.
\end{abstract}

PACS numbers: 03.67.Mn, 03.65.Ud, 03.65.Yz

\section{INTRODUCTION}

Atomic ensemble can serve as a quantum memory for storing the quantum information of photons [1] $[4]$ where the memory elements can be described as the quasi-spin wave excitations in atomic ensemble [5], or Dicke type collective states [6]. Recent experiments have demonstrated that such storable and quantum memory could have long lifetime for the use in long-distance quantum communication [7]. When using the magnetically insensitive clock transition in atomic rubidium confined in a one-dimensional optical lattice, quantum memory lifetime can exceed $3 \mathrm{~ms}$ [8].

The relevant quantum storage process could be implemented through the many-particle enhancement of the absorption cross section with the adiabatic-passage techniques [9]. Through the electromagnetically induced transparency (EIT) mechanism, the adiabatic manipulation utilizes the dark polariton $\left|d_{n}(\theta)\right\rangle$ (a photon-dressed collective atomic state). With adiabatically changing parameter $\theta \in[0, \pi / 2]$, the quantum state of photon $|P\rangle=\sum_{n} c_{n}|n\rangle$ (a superposition of photon Fock states $|n\rangle)$ can be adiabatically converted to a state of the collective atom excitation $|M\rangle=\sum_{n} c_{n}\left|M_{n}\right\rangle$, which is a superposition of the collective atomic states $\left|M_{n}\right\rangle$ with the same coefficients $c_{n}$ as that in the photon state $|P\rangle$. Mathematically, this coherent conversion of the photon state is an associated mapping

$$
|P\rangle \otimes\left|d^{\prime}\right\rangle=\sum c_{n}\left|d_{n}(0)\right\rangle \rightarrow \sum c_{n}\left|d_{n}\left(\frac{\pi}{2}\right)\right\rangle=\left|p^{\prime}\right\rangle \otimes|M\rangle
$$

where $\left|d^{\prime}\right\rangle$ and $\left|p^{\prime}\right\rangle$ are the initial state of the memory and the final state of photon respectively. Obviously, here only used are the macroscopically coherent properties of each collective excitation, which is described by individual collective state $\left|M_{n}\right\rangle$, rather than the internal entanglement and quantum correlation in the single collective state $\left|M_{n}\right\rangle$ as well as $\left|d_{n}(\theta)\right\rangle$.

In this paper we will pay attention to the latter by considering the multipartite correlation measured by spin squeezing in $\left|M_{n}\right\rangle$, which is obtained from $\left|d_{n}(\theta)\right\rangle$ by adiabatic manipulation for the dark state $\left|d_{n}(\theta)\right\rangle$ as

$$
|n\rangle \otimes|0\rangle=\left|d_{n}(0)\right\rangle \rightarrow\left|d_{n}\left(\frac{\pi}{2}\right)\right\rangle=|0\rangle \otimes\left|M_{n}\right\rangle .
$$

We study in details the dynamic competition between the adiabatic creation of quantum correlation in the atomic ensemble and the decoherence that we describe with three typical decoherence channels. There are two time scales $t_{1}$ and $t_{2}$ depicting such competition, where $t_{1}$ represents the adiabatic time limited by the adiabatic conditions and $t_{2}$ represents the decoherence time. Actually, if we regard the atomic ensemble as a macroscopic object in large $\mathrm{N}$ limit, quantum decoherence scenario seems to result in the quantum disappearance due to noisy ambient environments [10] or the internal random motions [11, 12]. A surprising discovery about the pairwise entanglement is that it suddenly die due to influence of environment [13, 14]. In this paper, we also consider this sudden death phenomena of the pairwise entanglement as well as the atomic squeezing for quantum correlation for more than two particles. We compare the time scales of various sudden death processes with the speed of adiabatic manipulations for creating such squeezing as well as the pairwise entanglement.

Actually, creating spin squeezing in a single Dicke like state is the crucial step for the precision measurements based on many-atom spectroscopy. With such controlled production of complex entangled states of matter and light, squeezing the fluctuations via entanglement between 2-level atoms can improve the precision of sensing, clocks, metrology, and spectroscopy [15]. This paper seems to provide a simple scheme for adiabatic creation of atomic squeezing. This adiabatic passage manipulation seems feasible in creating the spin squeezing, but it requires an ideal technique of single photon source, which can initially produce the single Fock states $|n\rangle$. Therefore, our present theoretical proposal may meet the difficulty for physical implementation based on the existing technology.

This paper is arranged as follows. In Sec. [I] we study 


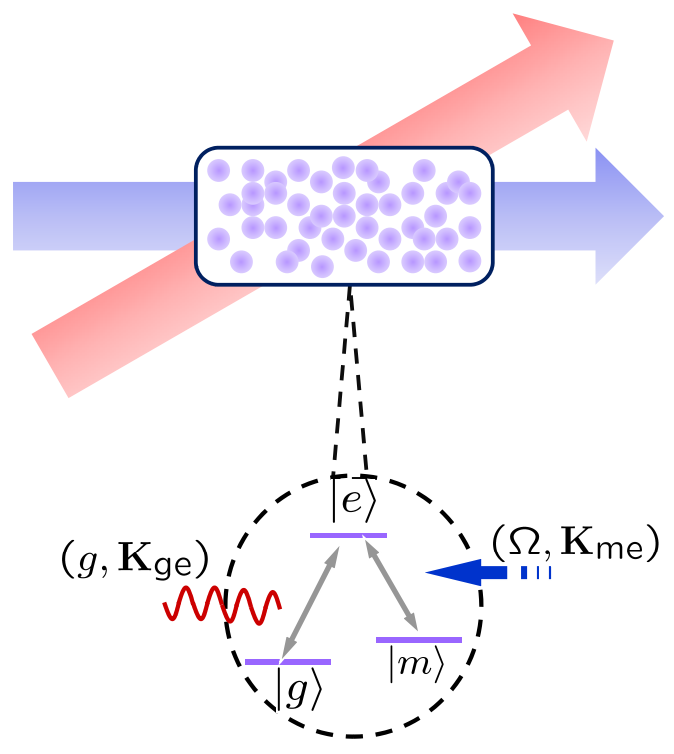

FIG. 1: (Color online) Schematic illustration of the atomic ensemble interacting with two light fields: the classical light field (denoted by the blue arrow) with Rabi frequency $\Omega$ and the wave vector $K_{\text {me }}$ and the quantum light field (denoted by the red arrow) with the coupling constant $g$ and the wave vector $K_{\text {ge }}$. Each $\Lambda$-type atom confined in the rectangular container has the same excited state $|e\rangle$, the relative ground state $|g\rangle$ and the metastable state $|m\rangle$.

the multipartite correlations characterized by the atomic squeezing beyond the pairwise entanglement in the dark states. In Sec. III we study the relationship between the concurrence and the spin squeezing under adiabatic manipulation, and indicate that the photon statistics varies in the same way of the spin squeezing. In Sec. IV, the sudden death of the atomic squeezing and the pairwise entanglement are considered by introducing three typical decoherence channels. The optimal times for generating the atomic squeezing and the pairwise entanglement are obtained in Sec. V]. We conclude in Sec. V.

\section{ADIABATICALLY CREATING SQUEEZING IN DARK STATES}

\section{A. Dark states}

As shown in Fig. 1 the model we consider consists of atomic ensemble with $N \Lambda$-type atoms, which have the excited state $|e\rangle$, the relative ground state $|g\rangle$ and the metastable state $|m\rangle$. For convenience we assume all the atoms have the same energy spacings for the above three atomic states. For the cold atomic ensemble, the Doppler width broadening of the atoms can be depressed effectively. These atoms interact with two single-mode light fields: one is quantized radiation mode (with coupling constant $g$ and annihilation operator $a$ ) to lead the approximately resonant transition from $|e\rangle$ to $|g\rangle$; the other is an exact classical field with Rabi frequency $\Omega$ to lead the transition from $|e\rangle$ to $|m\rangle$. The quantum dynamics of the total system is described by the following Hamiltonian in the interaction picture [5]

$$
\begin{aligned}
& H=g a \sum_{\mathbf{j}=1}^{N} \exp \left(i \mathbf{K}_{\mathrm{ge}} \cdot \mathbf{j}\right) \sigma_{\mathrm{ge}}^{\mathbf{j}}+ \\
& \Omega \sum_{\mathbf{j}=1}^{N} \exp \left(i \mathbf{K}_{\mathrm{me}} \cdot \mathbf{j}\right) \sigma_{\mathrm{me}}^{\mathbf{j}}+h . c .
\end{aligned}
$$

where $\mathbf{K}_{\mathrm{ge}}$ and $\mathbf{K}_{\mathrm{me}}$ are, respectively, the wave vectors of the quantum and the classical light fields. We have introduced the quasi-spin operators $\sigma_{\alpha \beta}^{\mathbf{j}} \equiv|\alpha\rangle_{\mathbf{j} \mathbf{j}}\langle\beta|(\alpha, \beta=$ $e, g, m)$ for $\alpha \neq \beta$ in above Hamiltonian to describe the transitions among the levels of $|e\rangle,|g\rangle$ and $|m\rangle$.

For EIT, to find the degenerate class of the eigenstates $\left|d_{n}(\theta)\right\rangle$ satisfying

$$
H\left|d_{n}(\theta)\right\rangle=0
$$

we introduce the dark state polariton operator

$$
D^{\dagger}(\theta)=a^{\dagger} \cos \theta-C^{\dagger} \sin \theta,
$$

which obviously mix the electromagnetic field with creation operator $a^{\dagger}$ and the quasi-spin wave with corresponding operator

$$
C^{\dagger}=\frac{1}{\sqrt{N}} \sum_{\mathbf{j}=1}^{N} \exp (i \mathbf{K} \cdot \mathbf{j}) \sigma_{m g}^{\mathbf{j}} .
$$

The angle $\tan \theta=g \sqrt{n} / \Omega$ can be adiabatically manipulated in the quantum storage process, and $\mathbf{K}=\mathbf{K}_{\mathrm{ge}}-$ $\mathbf{K}_{\text {me }}$ is the wave vector difference between the quantum and the classical fields. We construct the degenerate class of the eigenstates $\left|d_{n}(\theta)\right\rangle$ as

$$
\left|d_{n}(\theta)\right\rangle=\frac{A(n, N, \theta)}{\sqrt{n !}}\left[D^{\dagger}(\theta)\right]^{n}|\mathbf{0}\rangle,
$$

where the normalization constant

$$
\begin{aligned}
A(n, N, \theta) & =\sqrt{\frac{(N-n) ! N^{n}}{N ! \sin ^{2 n} 2 \theta}} \times \\
& {\left[{ }_{1} F_{1}\left(-n ; N-n+1 ;-N \cot ^{2} \theta\right)\right]^{-\frac{1}{2}} }
\end{aligned}
$$

reduces to 1 when the atom number $N$ is much larger than $n$. Here, $n$ is the excitation number of the atomic ensemble, the vacuum state

$$
|\mathbf{0}\rangle=|0\rangle \otimes|\downarrow\rangle
$$

is the direct product of the photonic vacuum state $|0\rangle$ and the quasi-spin ground state $|\downarrow\rangle \equiv \prod_{\mathbf{j}=\mathbf{1}}^{N}|g\rangle_{\mathbf{j}}$. 
Here, ${ }_{1} F_{1}(a ; b ; c)$ is the Kummer hypergeometric function [16]. Eq. (4) indicates that $\left|d_{n}(\theta)\right\rangle$ is totally cancelled by the interaction Hamiltonian, and thus is called a dark state or a dark state polariton.

Obviously, the dark state $\left|d_{n}(\theta)\right\rangle$ is photon Fock state $|n\rangle$ when $\theta=0$ and quasi-spin wave state when $\theta=$ $\pi / 2$. In the low excitation limit as $n \ll N, C^{\dagger}$ is an approximately bosonic operator as well as $D^{\dagger}(\theta)$.

With the help of the dark states, by adiabatically manipulating the classical field the quantum information of the photon with quantum state $|P\rangle=\sum_{n} c_{n}|n\rangle$ can be coherently converted into the atomic ensemble as $|M\rangle=\sum_{n} c_{n}\left|M_{n}\right\rangle$, where $|n\rangle$ is the Fock state of photon and

$$
\begin{aligned}
\left|M_{n}\right\rangle & =\left|d_{n}\left(\theta=\frac{\pi}{2}\right)\right\rangle \\
& =(-1)^{n} \sqrt{\frac{N^{n}(N-n) !}{N ! n !}}\left[C^{\dagger}\right]^{n}|\mathbf{0}\rangle
\end{aligned}
$$

is the collective atomic state. This coherent conversion is an associated mapping

$$
|P\rangle \otimes|\downarrow\rangle=\sum c_{n}\left|d_{n}(0)\right\rangle \rightarrow \sum c_{n}\left|d_{n}\left(\frac{\pi}{2}\right)\right\rangle=|0\rangle \otimes|M\rangle,
$$

which only use the macroscopically coherent properties of each collective excitation described by individual collective state $\left|M_{n}\right\rangle$. We notice that $\left|M_{n}\right\rangle$ is actual multiatom state with multipartite quantum correlations. For two-particle case, we call it internal entanglement.

The internal entanglement and the quantum correlation in the single collective state $\left|M_{n}\right\rangle$ as well as $\left|d_{n}(\theta)\right\rangle$ also play important role in quantum storage process. Now we consider the quantum correlation measured by spin squeezing of those quasi-spins in $\left|M_{n}\right\rangle$, which is obtained from $\left|d_{n}(\theta)\right\rangle$ by adiabatic manipulation

$$
|n\rangle \otimes|0\rangle=\left|d_{n}(0)\right\rangle \rightarrow\left|d_{n}\left(\frac{\pi}{2}\right)\right\rangle=|0\rangle \otimes\left|M_{n}\right\rangle .
$$

\section{B. Atomic squeezing in dark states without decoherence}

The above dark state mixes the electromagnetic field and the quasi-spins defined by the two levels $|g\rangle$ and $|m\rangle$ of the atomic ensemble. The collective operators

$$
J_{\alpha}=\frac{1}{2} \sum_{\mathbf{j}=1}^{N} \sigma_{\alpha}^{\mathbf{j}},(\alpha=x, y, z)
$$

can be generally used to demonstrate the exchange symmetry and dynamic properties of the quasi-spin system. To calculate the averages of the collective operators, the reduced density matrix of the quasi-spins is obtained by tracing over the degree of freedom of the photons as

$$
\begin{aligned}
& \rho_{\mathrm{a}}^{\mathrm{r}}= \operatorname{Tr}_{\mathrm{p}}\left|d_{n}(\theta)\right\rangle\left\langle d_{n}(\theta)\right| \text { or } \\
& \rho_{\mathrm{a}}^{\mathrm{r}}=\sum_{k=0}^{n} \frac{n !(\cos \theta)^{2 k}(\sin \theta)^{2 n-2 k}}{k !(n-k) !} \frac{|A(n, N, \theta)|^{2} N !}{N^{k}(N-n+k) !} \times \\
& U\left|n-k-\frac{N}{2}\right\rangle\left\langle n-k-\frac{N}{2}\right| U^{\dagger}
\end{aligned}
$$

where

$$
\left|k-\frac{N}{2}\right\rangle \equiv \sqrt{\frac{(N-k) !}{N ! k !}}\left(J_{+}\right)^{k}|\downarrow\rangle
$$

is a symmetric Dicke state and the collective operator

$$
J_{+}=\sqrt{N} U^{\dagger} C^{\dagger} U
$$

is a unitary transformation of the quai-spin wave operator with the unitary matrix

$$
U=\exp \left[-i \sum_{j=1}^{N} \mathbf{K} \cdot \mathbf{j} \frac{\left(\sigma_{z}^{\mathbf{j}}+1\right)}{2}\right] .
$$

Since only is the $z$-component quasi-spin operator contained in the unitary transformation, which will never change the quantum number of the symmetric Dicke bases, the density matrix in Eq. (14) only has the diagonal elements. Thus the averages of $J_{x}$ and $J_{y}$ vanish as $\left\langle J_{x}\right\rangle=\left\langle J_{y}\right\rangle=0$. In this situation, only the $z$-component collective operator $J_{z}$ survives as

$$
\left\langle J_{z}\right\rangle=\left.\left\langle J_{z}\right\rangle\right|_{\theta=\frac{\pi}{2}}+\delta J_{z}
$$

where $\left.\left\langle J_{z}\right\rangle\right|_{\theta=\pi / 2}=n-N / 2$ is mean $z$-component spin of the Dicke state $|n-N / 2\rangle$ and

$$
\delta J_{z}=-\frac{n N \cot ^{2} \theta_{1} F_{1}\left(1-n ; N-n+2 ;-N \cot ^{2} \theta\right)}{(N-n+1)_{1} F_{1}\left(-n ; N-n+1 ;-N \cot ^{2} \theta\right)}
$$

is the deviation of the $z$-component spin. The fact that $\left\langle J_{z}\right\rangle$ does not vanish means that the mean spin is along the $z$-direction. Since $\delta J_{z}$ vanishes when $\theta=\pi / 2$, its value can measure the mixture of the electromagnetic field and the quasi-spin wave.

The spin squeezing has several measures, and we only list three typical and related ones as follows:[14, 17 -19]

$$
\begin{aligned}
\xi_{1}^{2} & =\frac{4\left(\Delta J_{\perp}\right)_{\min }^{2}}{N}, \\
\xi_{2}^{2} & =\frac{N^{2}}{4\left\langle\mathbf{J}^{2}\right\rangle} \xi_{1}^{2}, \\
\xi_{3}^{2} & =\frac{\lambda_{\min }}{\left\langle\mathbf{J}^{2}\right\rangle-\frac{N}{2}} .
\end{aligned}
$$

In Eq.(20a), the minimization is over all the direction denoted by $\perp$, which is perpendicular to the mean spin direction $\langle\mathbf{J}\rangle /\left\langle\mathbf{J}^{2}\right\rangle$. For the quasi-spins in the dark state, 
the $\xi_{1}^{2}$ and $\xi_{2}^{2}$ actually measure the spin squeezing of the atomic ensemble in the $x-y$ plane. In Eq.(20c), $\lambda_{\min }$ is the minimal eigenvalue of matrix

$$
\Gamma=(N-1) \gamma+\mathbf{C},
$$

where

$$
\gamma_{k l}=\mathbf{C}_{k l}-\left\langle J_{k}\right\rangle\left\langle J_{l}\right\rangle(k, l \in\{x, y, z\})
$$

is the covariance matrix and

$$
\mathbf{C}_{k l}=\frac{1}{2}\left\langle J_{l} J_{k}+J_{k} J_{l}\right\rangle
$$

is the global correlation matrix. Parameters $\xi_{1}^{2}, \xi_{2}^{2}$, and $\xi_{3}^{2}$ were defined by Kitagawa and Ueda, Wineland et al., and Toth et al., respectively. If $\xi_{2}^{2}<1\left(\xi_{3}^{2}<1\right)$, the spin squeezing occurs, and we can safely say that the multipartite state is entangled [19, 20]. Although we cannot say that the squeezed state according to $\xi_{1}^{2}<1$ is entangled, it is indeed closely related to quantum entanglement [21].

For the quasi-spins in the dark state, since the averages $\left\langle J_{x} J_{z}\right\rangle=\left\langle J_{y} J_{z}\right\rangle=0$ vanish as the same reason of the vanishing of $\left\langle J_{x}\right\rangle$ and $\left\langle J_{y}\right\rangle$, the $\xi_{1}^{2}$ and $\xi_{3}^{2}$ are simplified as 14,21 ]

$$
\begin{aligned}
\xi_{1}^{2} & =\frac{2}{N}\left(\left\langle J_{x}^{2}+J_{y}^{2}\right\rangle-\left|\left\langle J_{-}^{2}\right\rangle\right|\right), \\
\xi_{3}^{2} & =\frac{\min \left\{\xi_{1}^{2}, \varsigma^{2}\right\}}{\frac{4}{N^{2}}\left\langle\mathbf{J}^{2}\right\rangle-\frac{2}{N}}
\end{aligned}
$$

where

$$
\varsigma^{2}=\frac{4}{N^{2}}\left[N\left(\Delta J_{z}\right)^{2}+\left\langle J_{z}\right\rangle^{2}\right]
$$

characterizes the spin squeezing along the $z$-direction. Because $\left\langle J_{-}^{2}\right\rangle=0$ for the quasi-spins in the dark state, $\xi_{1}^{2}$ and $\xi_{3}^{2}$ are always greater than 1 and thus there is no spin squeezing of the atomic ensemble in the $x-y$ plane. In the following discussions, we only use

$$
\xi_{3}^{2}=\frac{\varsigma^{2}}{\frac{4}{N^{2}}\left\langle\mathbf{J}^{2}\right\rangle-\frac{2}{N}}
$$

to characterize the $z$-component spin squeezing of the atomic ensemble in the dark states.

We consider the special case that when the wave vector difference $\mathbf{K}$ between the quantum and the classical field is zero. Since $\left.U\right|_{\mathbf{K}=0}$ is identity matrix, the quai-spin wave operator in Eq. (16) actually is proportional to the collective operator and the reduced density matrix in Eq. (14) is spanned by the symmetric Dicke states with total quasi-spin $\mathbf{J}=N / 2$. Thus

$$
\left.\left\langle\mathbf{J}^{2}\right\rangle\right|_{\mathbf{K}=0}=\frac{N}{2}\left(\frac{N}{2}+1\right),
$$

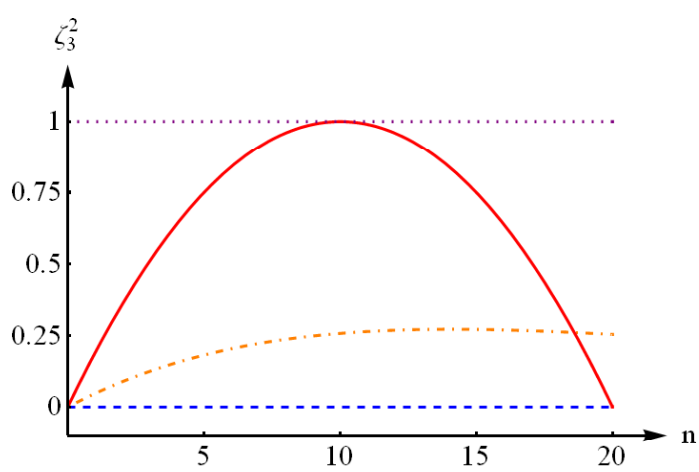

FIG. 2: (Color online) The squeezing parameter $\zeta_{3}^{2}$ versus the excitation number $n$ for $\theta=0$ (blue dashed line), $\theta=\pi / 4$ (orange dotdashed line) and $\theta=\pi / 2$ (red solid line). The total number of the atoms $N$ are chosen as 20. The horizontal purple dotted line as the base line represents the upper limit of the spin-squeezing. The dark state is always squeezed except without excitation $(n=0)$ or fully excited $(n=N)$.

and the straightforward calculation gives

$$
\begin{aligned}
\left.\xi_{3}^{2}\right|_{\mathbf{K}=0} & =\varsigma^{2}=\frac{4}{N^{2}}\left[N\left\langle J_{z}^{2}\right\rangle+(1-N)\left\langle J_{z}\right\rangle^{2}\right], \\
\left\langle J_{z}^{2}\right\rangle & =\left.\left\langle J_{z}^{2}\right\rangle\right|_{\theta=\frac{\pi}{2}}+\delta J_{z}^{2} \\
\delta J_{z}^{2} & =N \cot ^{2} \theta\left[2 n+N \cot ^{2} \theta\right. \\
& \left.-\frac{(N+1)\left(n+N \cot ^{2} \theta\right)}{(N-n+1)} \Gamma(n, N, \theta)\right]
\end{aligned}
$$

where

$$
\left.\left\langle J_{z}^{2}\right\rangle\right|_{\theta=\frac{\pi}{2}}=\left(n-\frac{N}{2}\right)^{2}
$$

is mean $z$-component spin square of the Dicke state $|n-N / 2\rangle$ and

$$
\Gamma(n, N, \theta)=\frac{{ }_{1} F_{1}\left(-n ; N-n+2 ;-N \cot ^{2} \theta\right)}{{ }_{1} F_{1}\left(-n ; N-n+1 ;-N \cot ^{2} \theta\right)} .
$$

For convenience we define equivalent squeezing parameter [14]

$$
\zeta_{3}^{2}=\max \left\{1-\xi_{3}^{2}, 0\right\},
$$

which characterizes the spin squeezing when $0<\zeta_{3}^{2} \leq 1$.

\section{Numerical pictures of the atomic squeezing in dark states}

The squeezing parameters of the dark state $\left|d_{n}(\theta)\right\rangle$ without wave vector difference $(\mathbf{K}=0)$ are illustrated in Fig. 2, 3 and 4. In all three figures, the total number of the atoms $N$ are chosen as 20. Since the reduced density matrix in Eq. (14) is symmetric when $\theta$ is replaced by $\theta+\pi$, the range of the $\theta$ is chosen as $[0, \pi)$. Fig. 2 shows 


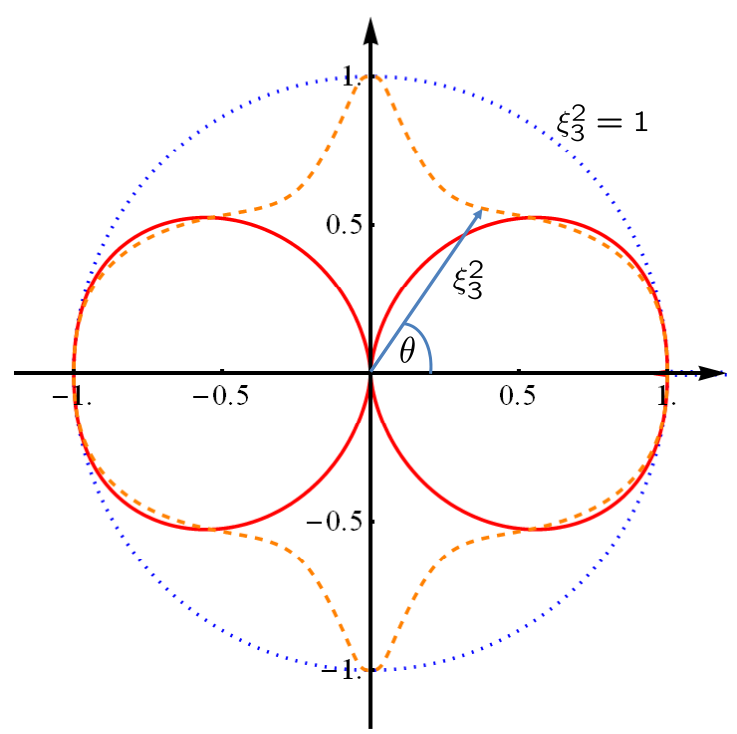

FIG. 3: (Color online) The polar plot of the squeezing parameter $\xi_{3}^{2}$ versus the parameter $\theta$ for the excitation number $n=0$ (blue dotted line), $n=10$ (orange dashed line) and $n=20$ (red solid line). The total number of the atoms $N$ are chosen as 20. The greatest spin-squeezing is obtained for the symmetric Dicke state when the half of the quasi-spins are excited $(n=N / 2)$ and $\theta=\pi / 2$.

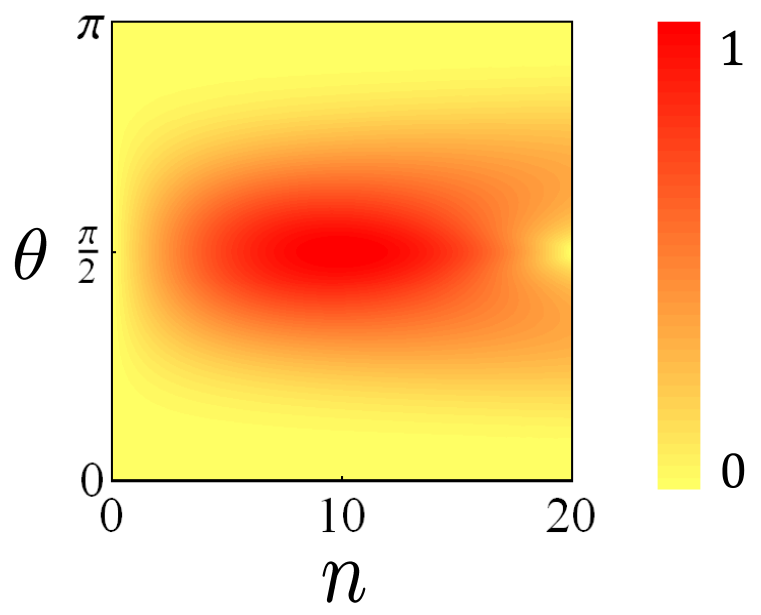

FIG. 4: (Color online) The contour plot of the squeezing parameter $\xi_{3}^{2}$ versus the excitation number $n$ and parameter $\theta$. The total number of the atoms $N$ are chosen as 20. The greatest spin-squeezing $\left(\zeta_{3}^{2}=1\right)$ is obtained for the symmetric Dicke state when the half of the quasi-spins are excited $(n=N / 2)$ and $\theta=\pi / 2$.

that the squeezing parameter $\zeta_{3}^{2}$ varies with the excitation number $n$ for different $\theta$. The dark state is always squeezed except without excitation $(n=0)$ or fully excited $(n=N)$. It is noticed that when $\theta \neq 0, \pi / 2$, the dark state is squeezed even when the excitation number $n$ is equal to the total number of the atoms $N$. Because the dark state actually mix the quasi-spins wave state

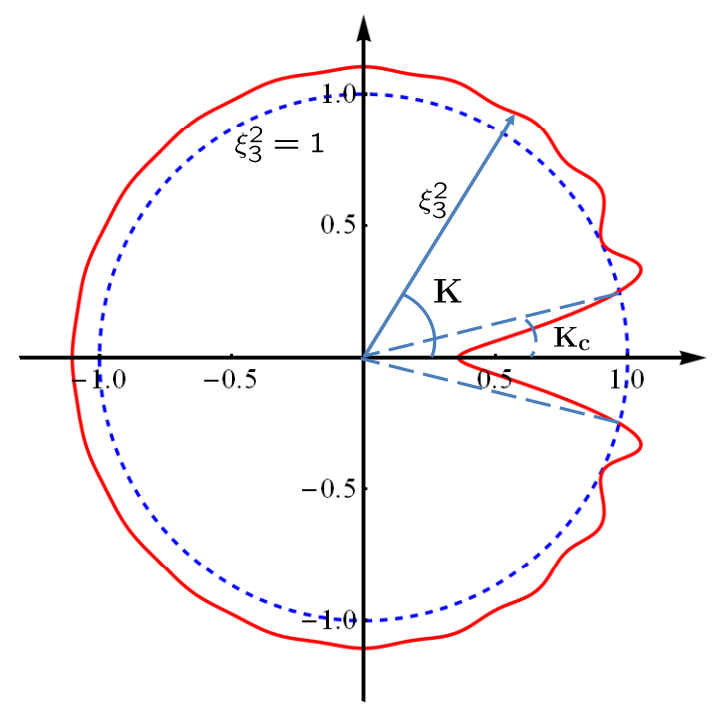

FIG. 5: (Color online) The polar plot of the squeezing parameter $\xi_{3}^{2}$ versus the vector difference $\mathbf{K}$. The total number of the atoms $N$, the excitation number $n$ and the parameter $\theta$ are chosen as 20,4 and $\pi / 2$. Here, $\xi_{3}^{2}=1$ is the baseline to measure whether the dark state is squeezed or not. Obviously, the dark state is squeezed only when the vector difference $\mathbf{K} \in\left[-\mathbf{K}_{\mathbf{c}}, \mathbf{K}_{\mathbf{c}}\right]$.

and the photon Fock state, this non-vanishing squeezing mainly results from the sub-Poisson distribution of the photons, which will be explicitly demonstrated in the last subsection of the Sec. II] Fig. 3 is the polar plot of the squeezing parameter $\rho=\xi_{3}^{2}(\theta)$ versus $\theta$ for different excitation numbers $n$. For the state without excitation $(n=0)$, the atomic ensemble always has no spin-squeezing because all of the quasi-spins are in their ground states. The greatest spin-squeezing is obtained for the symmetric Dicke state when the half of the quasispins are excited $(n=N / 2)$ and $\theta=\pi / 2$. Fig. 目 is the contour plot of the squeezing parameter $\zeta_{3}^{2}$ versus the excitation number $n$ and parameter $\theta$. Obviously, the greatest spin-squeezing ( $\zeta_{3}^{2}=1$ drawn by red color) can be obtained by adiabatically manipulating the parameter $\theta$ to $\pi / 2$ at the half excitation of the quasi-spins $(n=N / 2)$.

If the wave vectors between the quantum and the classical field are different, the nonzero $\mathbf{K}$ results in the nonsymmetrical Dicke bases in Eq. (14) and the spin squeezing decreases rapidly as the $\mathbf{K}$ deviates from 0 . Fig. 5 is the polar plot of the squeezing parameter $\rho=\xi_{3}^{2}(\theta)$ versus $\mathbf{K}$. The parameters are chosen as $N=20, n=4$, and $\theta=\pi / 2$. Since $\xi_{3}^{2}=1$ is the baseline to measure the atomic squeezing, the dark state is squeezed only when the vector difference $\mathbf{K} \in\left[-\mathbf{K}_{\mathbf{c}}, \mathbf{K}_{\mathbf{c}}\right]$, where $\mathbf{K}_{\mathbf{c}}$ is shown in Fig. 5 Fig. 6 is the contour plot of the squeezing parameter $\zeta_{3}^{2}$ versus the vector difference $\mathbf{K}$ and parameter $\theta$. The total number of the atoms $N$ and the excitation number $n$ are chosen as 20,4. In Fig. 6. $\left[-\mathbf{K}_{\mathbf{c}}, \mathbf{K}_{\mathbf{c}}\right]$ is still 


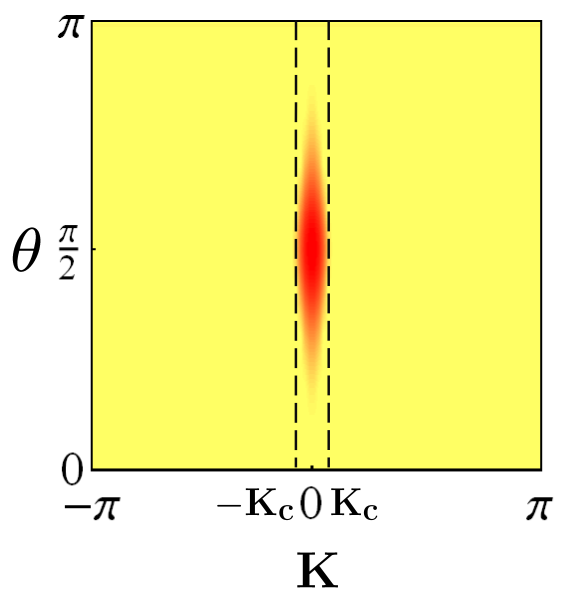

FIG. 6: (Color online) The contour plot of the squeezing parameter $\xi_{3}^{2}$ versus the vector difference $\mathbf{K}$ and the parameter $\theta$. The total number of the atoms $N$ and the excitation number $n$ are chosen as 20,4. Obviously, the dark state is squeezed only when the vector difference $\mathbf{K} \in\left[-\mathbf{K}_{\mathbf{c}}, \mathbf{K}_{\mathbf{c}}\right]$.

the boundary for the vector difference $K$ when the dark states are squeezed.

\section{INTERNAL ENTANGLEMENT IN DARK STATES AND PHOTON STATISTICS}

\section{A. Concurrence in dark states without decoherence}

As the physical observable to quantify the pairwise entanglement of spin- $1 / 2$, the concurrence is closely related to spin squeezing [21]. The concurrence is defined as [22]

$$
C=\max \left\{0, \lambda_{1}-\lambda_{2}-\lambda_{3}-\lambda_{4}\right\}
$$

where the quantities $\lambda_{i}(i=1,2,3,4)$ are the square roots of the eigen-values in descending order of the matrix product

$$
\varrho_{12}=\rho_{12}\left(\sigma_{1 y} \otimes \sigma_{2 y}\right) \rho_{12}^{*}\left(\sigma_{1 y} \otimes \sigma_{2 y}\right) .
$$

Here, $\rho_{12}$ is the two-spin reduced density matrix and $\rho_{12}^{*}$ is the complex conjugate of the $\rho_{12}$.

For the dark states, the two-quasi-spin reduced density matrix 23]

$$
\rho_{12}=\left[\begin{array}{cccc}
v_{+} & 0 & 0 & u^{*} \\
0 & w & y & 0 \\
0 & y & w & 0 \\
u & 0 & 0 & v_{-}
\end{array}\right]
$$

can be explicitly obtained by tracing out the degrees of freedom of all the other quasi-spins. It is clear to verify that for the dark states only the elements $v_{ \pm}, u, w$ and $y$ of the $\rho_{12}$ survive as $(\phi=\mathbf{K} \cdot \mathbf{l})$

$$
\begin{aligned}
v_{ \pm} & =\left.v_{ \pm}\right|_{\theta=\frac{\pi}{2}}+\delta v_{ \pm}, \\
w & =\left.w\right|_{\theta=\frac{\pi}{2}}+\delta w, \\
u & =-w i \sin \phi \\
y & =w \cos \phi
\end{aligned}
$$

where [23]

$$
\begin{aligned}
\left.v_{+}\right|_{\theta=\frac{\pi}{2}} & =\frac{1}{4}\left(1+2\left\langle\sigma_{z}^{1}\right\rangle+\left\langle\sigma_{z}^{1} \sigma_{z}^{2}\right\rangle\right) \\
& =\frac{n(n-1)}{N(N-1)} \\
\left.v_{-}\right|_{\theta=\frac{\pi}{2}} & =\frac{1}{4}\left(1-2\left\langle\sigma_{z}^{1}\right\rangle+\left\langle\sigma_{z}^{1} \sigma_{z}^{2}\right\rangle\right) \\
& =\frac{(n-N)(n-N+1)}{N(N-1)}, \\
\left.w\right|_{\theta=\frac{\pi}{2}} & =\frac{1}{4}\left(1-\left\langle\sigma_{z}^{1} \sigma_{z}^{2}\right\rangle\right) \\
& =\frac{n N-n^{2}}{N(N-1)}
\end{aligned}
$$

are the corresponding elements of the reduced density matrix over the Dicke state $|n-N / 2\rangle$ and

$$
\begin{gathered}
\delta v_{+}=B(N, \theta)\left[n+\left(N \csc ^{2} \theta+n-1\right) \Lambda(n, N, \theta)\right], \\
\delta v_{-}=B(N, \theta)\left[n+\left(N \cot ^{2} \theta-N+2 n+1\right) \Lambda(n, N, \theta)\right], \\
\delta w=B(N, \theta)\left[-n-\left(n+N \cot ^{2} \theta\right) \Lambda(n, N, \theta)\right]
\end{gathered}
$$

with $B(N, \theta)=\cot ^{2} \theta /(N-1)$ and

$$
\Lambda(n, N, \theta)=1-\frac{(N+1)}{(N-n+1)} \Gamma(n, N, \theta),
$$

where $\Gamma(n, N, \theta)$ is defined in Eq. (30).

Thus the concurrence of the dark state is given by

$$
C=\max \left\{0,2\left(w|\cos \phi|-\sqrt{v_{+} v_{-}}\right)\right\} .
$$

Fig. [7 is the contour plot of the concurrence $C$ versus the vector difference $\mathbf{K}$ and the parameter $\theta$. In comparison with the one region $\left[-\mathbf{K}_{\mathbf{c}}, \mathbf{K}_{\mathbf{c}}\right]$ for generating atomic squeezing, there are three regions for generating pairwise entanglement. This is also shown in Fig. 8, which is the sections of the atomic squeezing and the concurrence. Obviously, in the vicinity of the $K=\pi$, the concurrence appears but atomic squeezing does not. In Fig. 7 and 8 , the total number of the atoms $N$ and the excitation number $n$ are chosen as 20,4. In the next section, the evolvement of the concurrence as well as the spin-squeezing under various decoherence channels will be considered.

\section{B. Sub-Poisson distribution of the photons}

We have shown that the atomic squeezing for information storage can be generated through the adiabatically manipulating the angle $\theta$. In another aspect, as 


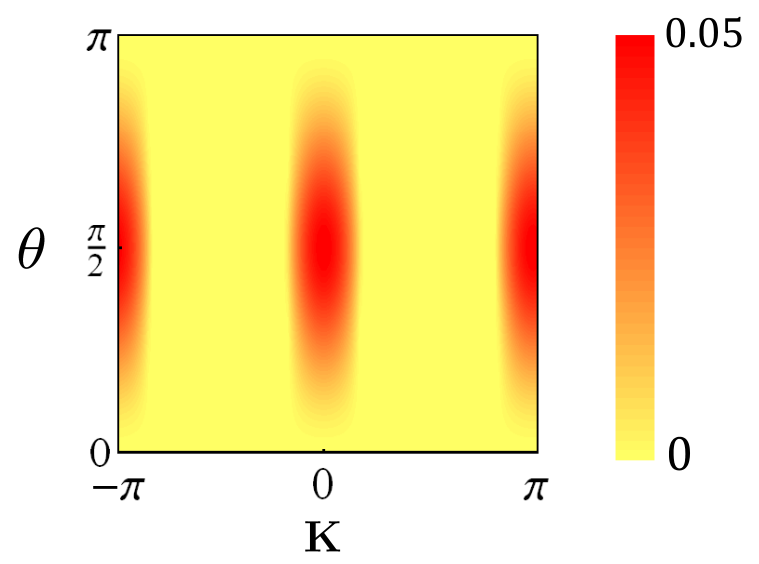

FIG. 7: (Color online) The contour plot of the concurrence $C$ versus the vector difference $\mathbf{K}$ and the parameter $\theta$. The total number of the atoms $N$ and the excitation number $n$ are chosen as 20 and 4 . Here we find three regions for generating concurrence rather than one region for generating atomic squeezing.

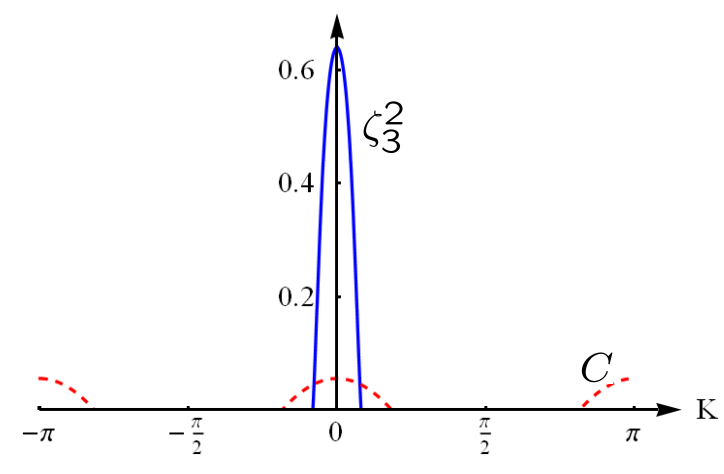

FIG. 8: (Color online) The sections of the atomic squeezing $\zeta_{3}^{2}$ (blue solid blue) and concurrence $C$ (red dashed line) versus the vector difference $\mathbf{K}$. The total number of the atoms $N$, the excitation number $n$ and the parameter $\theta$ are chosen as 20,4 and $\pi / 2$. Obviously, in the vicinity of the $\mathbf{K}=\pi$, the concurrence appears but atomic squeezing does not.

the photon dressed collective atom state the dark states mix the atomic ensemble state and the photon states. In our setup, the conservative quantity is the summation of the $z$-component collective operator $J_{z}$ and the number operator $n=a^{\dagger} a$ of the photons. The multipartite quantum correlation in dark states actually is the $z$-component spin squeezing for the dark states, which would result from the quantum correlation of the photons. Usually, such photonic quantum correlation can be measured by the sub-Poisson distribution of the photons

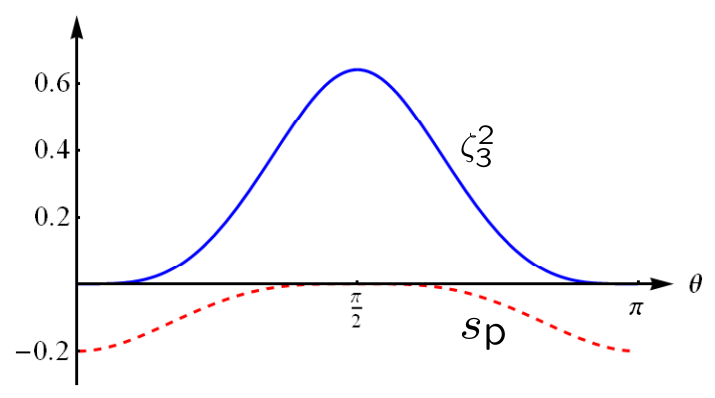

FIG. 9: (Color online) The sections of the atomic squeezing $\zeta_{3}^{2}$ (blue solid blue) and sub-Poisson distribution $s_{\mathbf{p}}$ (red dashed line) versus the parameter $\theta$. The total number of the atoms $N$, the excitation number $n$ and the vector difference $K$ are chosen as 20,4 and 0 . When the $\theta$ increases from 0 to $\pi / 2$, the atomic squeezing increases and the sub-Poisson distribution decreases simultaneously and vise vesa when the $\theta$ increases from $\pi / 2$ to $\pi$.

defined as

$$
\begin{aligned}
s_{\mathrm{p}} & =\frac{1}{N}\left(\left\langle\Delta n^{2}\right\rangle-\langle n\rangle\right) \\
& =\frac{1}{N}\left(\left\langle a^{\dagger} a^{\dagger} a a\right\rangle-\left\langle a^{\dagger} a\right\rangle^{2}\right) .
\end{aligned}
$$

If the photonic number fluctuation

$$
\left\langle\Delta n^{2}\right\rangle=\left\langle n^{2}\right\rangle-\langle n\rangle^{2}
$$

is smaller than the average of the particle number $\langle n\rangle$ of the photons $\left(s_{\mathrm{p}}<0\right)$, the dark states actually has the sub-Poisson distribution.

The straightforward calculation gives

$$
\begin{aligned}
&\left\langle a^{\dagger} a^{\dagger} a a\right\rangle=\frac{(n-1) n N^{2} \cot ^{4} \theta}{(N-n+2)(N-n+1)} \times \\
& \frac{{ }_{1} F_{1}\left(2-n ; N-n+3 ;-N \cot ^{2} \theta\right)}{{ }_{1} F_{1}\left(-n ; N-n+1 ;-N \cot ^{2} \theta\right)}, \\
&\left\langle a^{\dagger} a\right\rangle=\frac{n N \cot ^{2} \theta}{(N-n+1)} \times \\
& \frac{{ }_{1} F_{1}\left(1-n ; N-n+2 ;-N \cot ^{2} \theta\right)}{{ }_{1} F_{1}\left(-n ; N-n+1 ;-N \cot ^{2} \theta\right)} .
\end{aligned}
$$

Substituting the relevant expectation values into Eqs. (40) leads to the explicit expression of the sub-Poisson distribution of the photons $s_{\mathrm{p}}$, which always follow the atomic squeezing $\zeta_{3}^{2}$. Fig. 9illustrates the numerical relationship between sub-Poisson distribution and the atomic squeezing. When the $\theta$ increases from 0 to $\pi / 2$, the atomic squeezing increases and the sub-Poisson distribution decreases simultaneously and vise vesa when the $\theta$ increases from $\pi / 2$ to $\pi$. From the above discussion, the greatest atomic squeezing and the concurrence can be generated when the parameter $\theta$ is manipulated to $\pi / 2$, which means all the sub-Poisson distribution of the photons converts to the atomic squeezing. 
In this section, we just consider the adiabatic manipulation without any decoherence process. In the next section, we will take the typical three atomic decoherence channels into consideration to estimate the sudden death of the atomic squeezing and concurrence. Since the coherence time of photons is much larger than atomic one, the photonic decoherence such as the dephasing due to the imperfect single-photon indistinguishability [24] is not considered below.

\section{ATOMIC SQUEEZING AND PAIRWISE ENTANGLEMENT UNDER DECOHERENCE CHANNELS}

\section{A. Decoherence channels}

Through the adiabatically manipulating the angle $\theta$, the spin-squeezing and the concurrence are generated for information storage. However, due to the existence of the environment, they are irreversible depressed and vanish eventually. Such decoherence processes are usually described by three typical decoherence channels: the amplitude damping channel (ADC), the phase damping channel (PDC) and the depolarizing channel (DPC) [21, 25]. They are defined by some maps of the elements of the density matrix $|i\rangle\langle j|$. Since the ADC is an energy-losing process and thus it forces the initial state to dissipate into the ground state, the map is defined as

$$
\begin{aligned}
& |i\rangle\langle i|\rightarrow(1-p)| i\rangle\langle i|+p| 0\rangle\langle 0|, \\
& |i\rangle\left\langle j\left|\rightarrow(1-p)^{\frac{1}{2}}\right| i\right\rangle\langle j|,(i \neq j) .
\end{aligned}
$$

The PDC is phase-losing process and the corresponding map is described by

$$
|i\rangle\left\langle j\left|\rightarrow\left(1-p+p \delta_{i, j}\right)\right| i\right\rangle\langle j| .
$$

The DPC is polarization-losing process and the corresponding map is represented by

$$
|i\rangle\langle j|\rightarrow(1-p)| i\rangle\langle j|+p \delta_{i, j} \frac{\mathbf{I}}{2} .
$$

The behaviors of the spin-squeezing and the concurrence under these decoherence channels will be present in the following sub sections. Here, $p$ is the decoherence strength whose range is $[0,1]$. When $p=0$ there is no decoherence and when $p=1$ the decoherence processes are completed.

If we fix the initial values of the spin-squeezing and the concurrence of the dark state, the dark state degrades to a product state with single quasi-spin state as a component when the decoherence strength $p$ increases from 0 . Thus the spin-squeezing and the concurrence would have sudden death. Before the sudden death happens, the information storage can be in progress safely. In other word, the longer time the spin-squeezing and the concurrence survive, the better the information storage can be achieved.
From the above expression of the spin-squeezing and the concurrence, we notice that if we know the expectations $\left\langle J_{z}\right\rangle,\left\langle J_{z}^{2}\right\rangle$ and $\left\langle\sigma_{z}^{1}\right\rangle$, and the correlations $\left\langle\sigma_{z}^{1} \sigma_{z}^{2}\right\rangle$, all the spin-squeezing and the concurrence can be determined. We will give explicit analytical expressions for them under three decoherence channels.

\section{B. Amplitude-damping channel}

Based on the map in Eq. (43) for ADC, one can find the following relations for single quasi-spin operators [14]

$$
\begin{aligned}
\left\langle\sigma_{z}^{1}(p)\right\rangle & =s\left\langle\sigma_{z}^{1}\right\rangle_{0}-p \\
\left\langle\sigma_{z}^{1} \sigma_{z}^{2}(p)\right\rangle & =s^{2}\left\langle\sigma_{z}^{1} \sigma_{z}^{2}\right\rangle_{0}-2 s p\left\langle\sigma_{z}^{1}\right\rangle_{0}+p^{2} \\
\left\langle\sigma_{\alpha}^{1} \sigma_{\beta}^{2}(p)\right\rangle & =s\left\langle\sigma_{\alpha}^{1} \sigma_{\beta}^{2}\right\rangle_{0}(\alpha, \beta=x, y)
\end{aligned}
$$

Hereafter, we define $s=1-p$. Then the corresponding relations for the collective operators are

$$
\begin{aligned}
\left\langle J_{z}^{2}(p)\right\rangle= & s^{2}\left\langle J_{z}^{2}\right\rangle_{0}+(N-1) s p\left\langle J_{z}\right\rangle_{0} \\
& +\frac{N^{2}}{4} p\left(p+\frac{2}{N} s\right) \\
\left\langle J_{z}(p)\right\rangle= & s\left\langle J_{z}\right\rangle_{0}-\frac{N}{2} p \\
\left\langle\mathbf{J}^{2}(p)\right\rangle= & s\left\langle\mathbf{J}^{2}\right\rangle_{0}-s p\left\langle J_{z}^{2}\right\rangle_{0}+(N-1) \operatorname{sp}\left\langle J_{z}\right\rangle_{0} \\
& +\frac{N}{4} p(N p+2 s) .
\end{aligned}
$$

Substituting the relevant expectation values and the correlation function into Eqs. (25/26) leads to the explicit expression of the spin-squeezing parameters

$$
\xi_{3}^{2}(p)^{\mathrm{A}}=\frac{\varsigma^{2}(p)}{\frac{4}{N^{2}}\left\langle\mathbf{J}^{2}(p)\right\rangle-\frac{2}{N}}
$$

with

$$
\varsigma^{2}(p)=s^{2} \varsigma_{0}^{2}+2 \frac{4}{N}(N-1) \operatorname{sp}\left\langle J_{z}\right\rangle_{0}+p(1+s),
$$

where $\varsigma_{0}^{2}$ is the initial spin-squeezing parameter defined in Eq. (25).

In the same case, in order to investigate the evolution of the concurrence, the two-qubit density matrix is shown as

$$
\rho_{12}^{\mathrm{A}}=\left[\begin{array}{cccc}
v_{+}^{\mathrm{A}} & 0 & 0 & u^{\mathrm{A}, *} \\
0 & w^{\mathrm{A}} & y^{\mathrm{A}} & 0 \\
0 & y^{\mathrm{A}} & w^{\mathrm{A}} & 0 \\
u^{\mathrm{A}} & 0 & 0 & v_{-}^{\mathrm{A}}
\end{array}\right]
$$

with $v_{+}^{\mathrm{A}}=s^{2} v_{+}, y^{\mathrm{A}}=s y, u^{\mathrm{A}}=s u$ and

$$
\begin{aligned}
v_{-}^{\mathrm{A}} & =s^{2} v_{+}-s\left\langle\sigma_{z}\right\rangle_{0}+p, \\
w^{\mathrm{A}} & =s^{2} w+\frac{1}{2} s p\left\langle\sigma_{z}^{1}\right\rangle_{0}+\frac{1}{2} s p,
\end{aligned}
$$




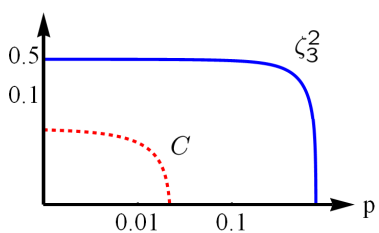

(a)

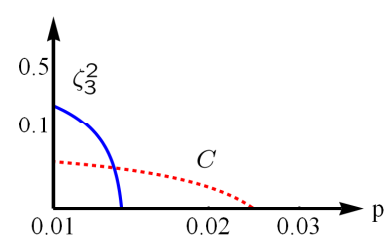

(b)

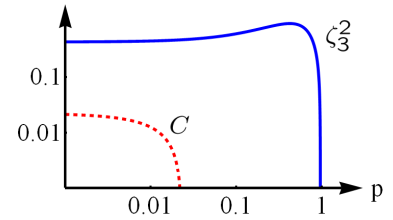

(c)
FIG. 10: (Color online) The sudden death of the atomic squeezing $\zeta_{3}^{2}$ (blue solid line) and the concurrence $C$ (red dashed line) under ADC. (a)Usually, the atomic squeezing $\zeta_{3}^{2}$ disappear later than the concurrence $C$. (b)The atomic squeezing $\zeta_{3}^{2}$ can disappear earlier than the concurrence $C$. (c)The atomic squeezing $\zeta_{3}^{2}$ may reach its maximum and then disappear near the maximum decoherence strength $p=1$. The parameters are chosen as $N=20, n=16, K=0$ and (a) $\theta=\pi / 3$, (b) $\theta=\pi / 2,(\mathrm{c}) \theta=0.673 \pi$.

where $v_{ \pm}, y, u$ and $w$ are initial elements defined in Eq. (35). Thus we obtain the time evolution of the concurrence as

$$
\begin{aligned}
& C^{\mathrm{A}}=\max \left\{0, C_{1}^{\mathrm{A}}, C_{2}^{\mathrm{A}}\right\}, \\
& C_{1}^{\mathrm{A}}=2\left(s w|\cos \phi|-\sqrt{v_{+}^{\mathrm{A}} v_{-}^{\mathrm{A}}}\right), \\
& C_{2}^{\mathrm{A}}=2\left(s w|\sin \phi|-w^{\mathrm{A}}\right) .
\end{aligned}
$$

Hereafter, we only consider the case at the vector difference $\mathrm{K}=0$ because the quantum correlations decrease rapidly when the vector difference deviates from 0 shown in Fig. 5 .

The subindex A in the above Eqs. 48,50,51,52) represents that the evolutions of the spin-squeezing and the concurrence are taken under ADC. The sudden death of the spin-squeezing and the concurrence implied in Eq. (48,52) are shown in Fig. 10. Usually, the atomic squeezing $\zeta_{3}^{2}$ disappear later than the concurrence $C$ as shown in Fig. 10(a). However for ADC, the atomic squeezing $\zeta_{3}^{2}$ can disappear earlier than the concurrence $C$ (Fig. 10(b)). With appropriate parameters, the atomic squeezing $\zeta_{3}^{2}$ may reach its maximum and then disappear near the maximum decoherence strength $p=1$ (Fig. 10(c)). The parameters are chosen as $N=20, n=$ $16, K=0$ and $(\mathrm{a}) \theta=\pi / 3,(\mathrm{~b}) \theta=\pi / 2,(\mathrm{c}) \theta=0.673 \pi$.

\section{Phase-damping channel}

For PDC described by the map in Eq. (44), one can

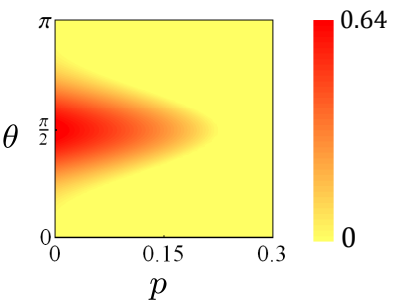

(a)

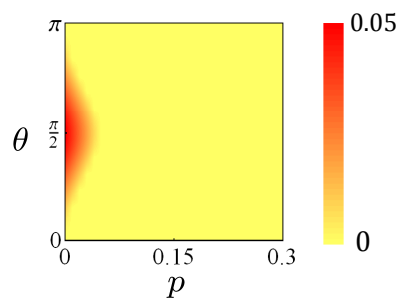

(b)
FIG. 11: (Color online) The sudden death of (a) the atomic squeezing $\zeta_{3}^{2}$ and (b) the concurrence $C$ under PDC. The parameters are chosen as $N=20, n=16$ and $K=0$. The atomic squeezing $\zeta_{3}^{2}$ always disappear later than the concurrence $C$.

find the following relations for single quasi-spin operators [14]

$$
\begin{aligned}
\left\langle\sigma_{z}(p)\right\rangle & =\left\langle\sigma_{z}\right\rangle_{0} \\
\left\langle\sigma_{z}^{1} \sigma_{z}^{2}(p)\right\rangle & =\left\langle\sigma_{z}^{1} \sigma_{z}^{2}\right\rangle_{0} \\
\left\langle\sigma_{\alpha}^{1} \sigma_{\beta}^{2}(p)\right\rangle & =s^{2}\left\langle\sigma_{\alpha}^{1} \sigma_{\beta}^{2}\right\rangle_{0}(\alpha, \beta=x, y) .
\end{aligned}
$$

Then the corresponding relations for the collective operators are $\left\langle J_{z}^{2}(p)\right\rangle=\left\langle J_{z}^{2}\right\rangle_{0},\left\langle J_{z}(p)\right\rangle=\left\langle J_{z}\right\rangle_{0}$ and

$$
\left\langle\mathbf{J}^{2}(p)\right\rangle=s^{2}\left\langle\mathbf{J}^{2}\right\rangle_{0}+\left(1-s^{2}\right) \frac{N}{2}
$$

Substituting the relevant expectation values and the correlation function into Eqs. (2526) leads to the explicit expression of the spin-squeezing parameters

$$
\xi_{3}^{2}(p)^{\mathrm{P}}=\frac{\varsigma_{0}^{2}}{\frac{4}{N^{2}}\left\langle\mathbf{J}^{2}(p)\right\rangle-\frac{2}{N}} .
$$

In the same sense to investigate the evolution of the concurrence, the two-qubit density matrix is present as

$$
\rho_{12}^{\mathrm{P}}=\left[\begin{array}{cccc}
v_{+}^{\mathrm{P}} & 0 & 0 & u^{\mathrm{P}, *} \\
0 & w^{\mathrm{P}} & y^{\mathrm{P}} & 0 \\
0 & y^{\mathrm{P}} & w^{\mathrm{P}} & 0 \\
u^{\mathrm{P}} & 0 & 0 & v_{-}^{\mathrm{P}}
\end{array}\right]
$$

with relations $v_{ \pm}^{\mathrm{P}}=v_{ \pm}, w^{\mathrm{P}}=w, y^{\mathrm{P}}=s^{2} y$, and $u^{\mathrm{P}}=$ $s^{2} u$. Thus we obtain the evolution of the concurrence as

$$
C^{\mathrm{P}}=\max \left\{0,2\left(s^{2} w|\cos \phi|-\sqrt{v_{+} v_{-}}\right)\right\} .
$$

The subindex $\mathrm{P}$ in the above Eqs. (55156157) represents that the time evolutions of the spin-squeezing and the concurrence are taken under PDC. The sudden death of the spin-squeezing and the concurrence implied in Eq. (55157) are shown in Fig. 11. In contrast of the ADC case, the atomic squeezing $\zeta_{3}^{2}$ always disappear later than the concurrence $C$. 


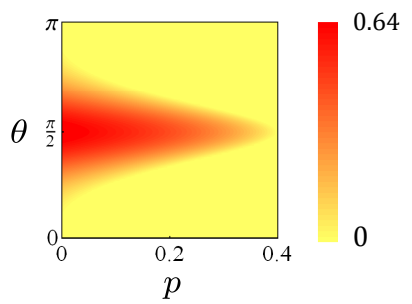

(a)

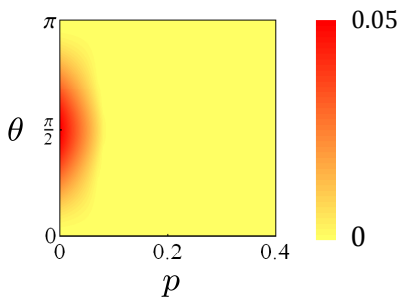

(b)
FIG. 12: (Color online) The sudden death of (a) the atomic squeezing $\zeta_{3}^{2}$ and (b) the concurrence $C$ under DPC. The parameters are chosen as $N=20, n=4$ and $K=0$. The atomic squeezing $\zeta_{3}^{2}$ always disappear later than the concurrence $C$.

\section{Depolarizing channel}

Based on the map in Eq. (45) for DPC, one can find the following relations for single quasi-spin operators [14]

$$
\begin{aligned}
\left\langle\sigma_{z}(p)\right\rangle & =s\left\langle\sigma_{z}\right\rangle_{0}, \\
\left\langle\sigma_{\alpha}^{1} \sigma_{\beta}^{2}(p)\right\rangle & =s^{2}\left\langle\sigma_{\alpha}^{1} \sigma_{\beta}^{2}\right\rangle_{0}(\alpha, \beta=x, y, z) .
\end{aligned}
$$

Then the corresponding relations for the collective operators are $\left\langle J_{z}(p)\right\rangle=s\left\langle J_{z}\right\rangle_{0}$ and

$$
\begin{aligned}
& \left\langle J_{z}^{2}(p)\right\rangle=s^{2}\left\langle J_{z}^{2}\right\rangle_{0}+\left(1-s^{2}\right) \frac{N}{4}, \\
& \left\langle\mathbf{J}^{2}(p)\right\rangle=s^{2}\left\langle\mathbf{J}^{2}\right\rangle_{0}+\left(1-s^{2}\right) \frac{3 N}{4} .
\end{aligned}
$$

Substituting the relevant expectation values and the correlation function into Eqs. (25I26) leads to the explicit expression of the spin-squeezing parameters

$$
\xi_{3}^{2}(p)^{\mathrm{D}}=\frac{s^{2} \varsigma_{0}^{2}+1-s^{2}}{\frac{4}{N^{2}}\left\langle\mathbf{J}^{2}(p)\right\rangle-\frac{2}{N}} .
$$

In the same sense to investigate the evolution of the concurrence, the two-qubit density matrix is present as

$$
\rho_{12}^{\mathrm{D}}=\left[\begin{array}{cccc}
v_{+}^{\mathrm{D}} & 0 & 0 & u^{\mathrm{D}, *} \\
0 & w^{\mathrm{D}} & y^{\mathrm{D}} & 0 \\
0 & y^{\mathrm{D}} & w^{\mathrm{D}} & 0 \\
u^{\mathrm{D}} & 0 & 0 & v_{-}^{\mathrm{D}}
\end{array}\right]
$$

with relations $y^{\mathrm{D}}=s^{2} y, u^{\mathrm{D}}=s^{2} u$ and

$$
\begin{aligned}
v_{ \pm}^{\mathrm{D}} & =\frac{s^{2}+s}{2} v_{ \pm}+\frac{s^{2}-s}{2} v_{\mp}+\frac{1-s^{2}}{4}, \\
w^{\mathrm{D}} & =s^{2} w_{0}+\frac{1-s^{2}}{4} .
\end{aligned}
$$

Thus we obtain the evolution of the concurrence as

$$
C^{\mathrm{D}}=\max \left\{0,2\left(s^{2} w|\cos \phi|-\sqrt{v_{+}^{\mathrm{D}} v_{-}^{\mathrm{D}}}\right)\right\} .
$$

The subindex D in the above Eqs. 601616363 represents that the evolutions of the spin-squeezing and the concurrence are taken under DPC. The sudden death of the spin-squeezing and the concurrence implied in Eq. (60)63) are shown in Fig. 12, In contrast of the ADC case, the atomic squeezing $\zeta_{3}^{2}$ always disappear later than the concurrence $C$.

\section{OPTIMAL TIME FOR GENERATING ATOMIC SQUEEZING}

Now, two competitive processes dominate generating the spin-squeezing. One is adiabatically manipulating the parameter $\theta$ from 0 to $\pi / 2$, which increases the spinsqueezing and the concurrence for storing the information of phonons into the atomic ensemble. However, the other process resulting from the various decoherence channels decreases the spin-squeezing and the concurrence, which means that the system loses information continuously. Therefore, the competition between this two processes leads to the existence of an optimal time for storing information, after which the information stored in the atomic ensemble is always losing. We can define two time scales: if only considering the adiabatic manipulation, one time scale is $t_{1}$ representing the time when the spin squeezing increases to the half maximum value; if only considering the decoherence processes, the other time scale is $t_{2}$ representing the time when the spin squeezing decreases to the half maximum value. Only when $t_{1}>t_{2}$ the optimal time exists.

We would like to demonstrate such competition in a typical quantum memory based on the cold ${ }^{87} \mathrm{Rb}$ atomic ensemble, which is released from a magnetooptical trap at a temperature of about $100 \mu K$ [7]. In such quantum memory, the ground state $|g\rangle$, the metastable state $|m\rangle$ and the excited state $|e\rangle$ are chosen as $\left|5 S_{1 / 2}, F=1, m_{F}=1\right\rangle,\left|5 S_{1 / 2}, F=2, m_{F}=-1\right\rangle$ and $\left|5 S_{1 / 2}, F=2, m_{F}=0\right\rangle$, respectively. An off-resonant $\sigma^{-}$-polarized write pulse contributes to the transition from $|g\rangle$ to $|e\rangle$ and the Stokes photon with $\sigma^{-}$polarization associates with the transition from $|e\rangle$ to $|m\rangle$. The dark state for storing the photonic information is described in Eq. (7).

To realize the adiabatic creation of the non-classical correlation, we adopt the hyperbolic tangent type pulse sequence for both Rabi frequencies [26] as

$$
\begin{aligned}
& g(t)=\Omega_{m}\left[1-\tanh \left(\frac{a}{t}+\frac{a}{t-\tau}\right)\right], \\
& \Omega(t)=\Omega_{m}\left[1+\tanh \left(\frac{a}{t}+\frac{a}{t-\tau}\right)\right]
\end{aligned}
$$

instead of the usual gaussian type ones [27] or solitonary type ones [28]. Here, $\Omega_{m}$ is the maximum Rabi frequency, $\tau$ is the pulse length and $a$ corresponds to the half width of the pulse. The pulse sequences of both Rabi frequencies are depicted in Fig. 13, Only at the time interval 


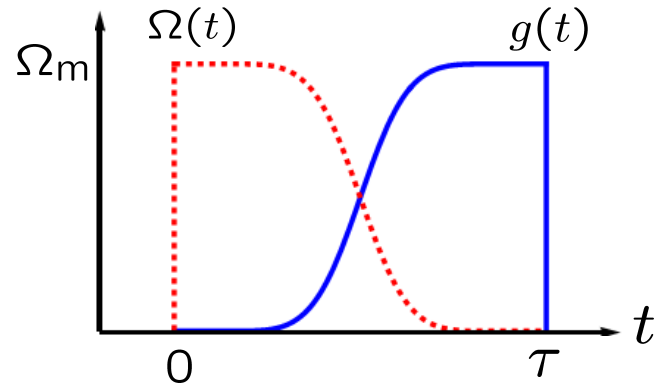

FIG. 13: (Color online) Optical pulse sequences for both Rabi frequencies. The red dashed line and black solid line represent the Rabi frequencies $\Omega(t)$ and $g(t)$, respectively. The adiabatic following condition is $\Omega_{m} \tau \gg 1$.

$[0, \tau]$ are both sequences applied to adiabatically manipulate the dark state in Eq. (77). Two Rabi frequencies respectively reach their maximum value $\Omega_{m}$ at the initial time $t=0$ and the terminal time $t=\tau$, which guarantees that the angle $\theta$ varies from 0 to $\pi / 2$ and thus the information of the photons is stored into the atomic ensemble at the time interval $[0, \tau]$.

Actually, due to the adiabatic manipulation, the time scale $t_{1}$ for generating spin squeezing is limited by the adiabatic condition

$$
\left|\frac{\left\langle n(t) \mid d_{n} \dot{(}(t)\right\rangle}{E_{n}-E_{d}}\right| \ll 1
$$

with eigenstates $|n(t)\rangle$ (the corresponding eigen-energy $\left.E_{n}\right)$ and the time-dependent dark states $\left|d_{n}(t)\right\rangle$ (the corresponding eigen-energy $E_{d}$ ). Here,

$$
\left\langle n(t) \mid d_{n}(t)\right\rangle \equiv \frac{\left\langle n(t)\left|\frac{\partial}{\partial t} H(t)\right| d_{n}(t)\right\rangle}{E_{n}-E_{d}}
$$

describes the variation due to the time-dependent Hamiltonian. Thus the condition for the adiabatic following is $\Omega_{m} \tau \gg 1$. The typical pulse length $\tau$ is about $150 \mu s[26]$, which means the maximum value of the Rabi frequencies $\Omega_{m}$ should much larger than $6.67 \times 10^{3} \mathrm{~Hz}$. According to the pulse sequence in Eq. (64), the time scale $t_{1}$ can be determined when $\theta\left(t_{1}\right)$ is tuned to $\pi / 6$ as

$$
t_{1}=\frac{2 a+\tau r-\sqrt{4 a^{2}+\tau^{2} r^{2}}}{2 r},
$$

where $r=\tanh ^{-1}[(6-\pi) /(6+\pi)]$. When $a$ tends to infinity, the longest time $t_{1}^{L} \simeq 1 / 2 \tau=75 \mu \mathrm{s}$ is obtained.

In the other hand, we also assume the exponential decays for the decoherence processes with the decoherence strength

$$
p(t)=1-e^{-\gamma t},
$$

which leads to the time scale

$$
t_{2}=\frac{1}{\gamma}
$$

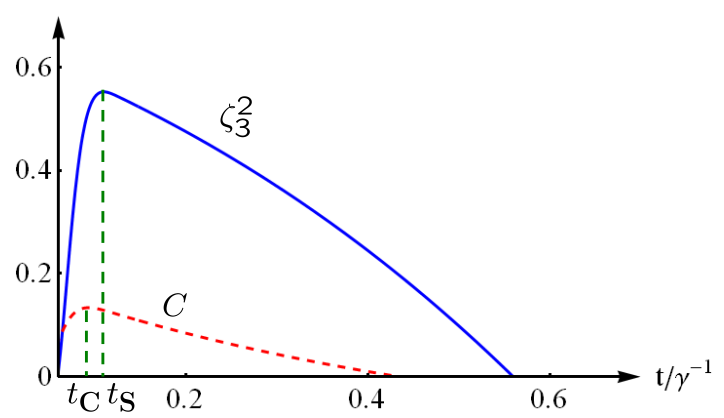

FIG. 14: (Color online) The evolution of the atomic squeezing $\zeta_{3}^{2}$ (blue solid line) and the concurrence $C$ (red dashed line). The parameters are chosen as $N=20, n=4, K=0$. The maximum of the atomic squeezing and the concurrence are respectively obtained at $t_{\mathrm{s}} \approx 0.12 \gamma^{-1}=120 \mu \mathrm{s}$ and $t_{\mathrm{c}} \approx 0.09 \gamma^{-1}=90 \mu \mathrm{s}$.

For example, for the cold trapped ${ }^{87} \mathrm{Rb}$ atomic ensemble, the typical dephasing damping rate $\gamma=10^{3} \mathrm{~Hz}$ and the corresponding dephasing time $t_{2}=1 \mathrm{~ms}[7,29]$.

The evolution of the spin-squeezing and the concurrence is shown in Fig. 14. Obviously, The maximum of the atomic squeezing and the concurrence are respectively obtained at $t_{\mathrm{s}} \approx 0.12 \gamma^{-1}=120 \mu \mathrm{s}$ and $t_{\mathrm{c}} \approx$ $0.09 \gamma^{-1}=90 \mu \mathrm{s}$. Though the Fig. 14 is drawn under PDC, the optimal times can also be obtained under ADC and DPC.

Such competition can also be observed by measuring the cross-correlation [7, 30]

$$
g_{S, A S}=1+C \Gamma(t)
$$

as a function of the time, where $C$ is a fitting parameter determined by the excitation probability together with the background noise in the anti-Stokes channel. Here,

$$
\Gamma(t)=\left|\left\langle\Psi_{\max } \mid \Psi(t)\right\rangle\right|^{2}
$$

is the retrieval efficiency, which actually characterizes the complete time-dependence properties of the crosscorrelation. For the dark state we discuss above, to estimate the adiabatical creation and the decoherence channels at the same time, we choose $\left|\Psi_{\max }\right\rangle$ as the collective atomic state $\left|M_{n}\right\rangle$ and thus the retrieval efficiency is obtained as

$$
\Gamma(t)=\frac{n !(N-n) !}{N !} \frac{{ }_{2} F_{1}\left(n-N,-n ; 1 ; e^{-2 \gamma t}\right)}{{ }_{1} F_{1}\left(-n ; N-n+1 ;-N \cot ^{2} \theta(t)\right)} .
$$

Here, ${ }_{2} F_{1}(a, b ; c ; z)$ is generalized Kummer hypergeometric function [16]. The retrieval efficiency is depicted in Fig. 15, in which the maximum of the retrieval efficiency exists when the same condition $t_{1}>t_{2}$ is satisfied. 


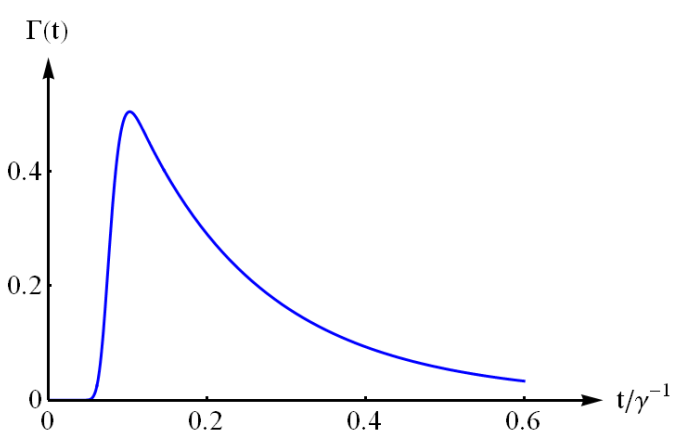

FIG. 15: (Color online). The retrieval efficiency as a function of the time. The parameters are chosen as $N=20, n=$ $4, K=0$. The maximum retrieval efficiency is also obtained around the $0.12 \gamma^{-1}=120 \mu \mathrm{s}$.

\section{CONCLUSION}

We investigate the multipartite correlations characterized by the atomic squeezing beyond the pairwise entanglement in the dark states, which are proposed for the quantum information storage based on electromagnetically induced transparency mechanism. The atomic squeezing and the pairwise entanglement can be created by adiabatically manipulating the Rabi frequency of the the classical light field on the atomic ensemble. The atomic squeezing in dark states converts from the sub-
Poisson distribution of the photons. The greatest atomic squeezing and the concurrence can be generated when the parameter $\theta$ is manipulated to $\pi / 2$, which means all the sub-Poisson distribution of the photons converts to the atomic squeezing.

We also consider the sudden death for the atomic squeezing and the pairwise entanglement under various decoherence channels. For the three typical decoherence channels, the sudden deaths of the atomic squeezing happens later than the sudden death of the concurrence.

According to the above investigation, an optimal time for generating the greatest atomic squeezing and pairwise entanglement is obtained by studying in details the competition between the adiabatic creation of quantum correlation in the atomic ensemble and the decoherences we describe with decoherence channels.

\section{Acknowledgments}

The work is supported by National Natural Science Foundation of China under Grant Nos. 10547101 and 10604002, the National Fundamental Research Program of China under Grant No. 2006CB921200. X. Wang is supported by NSFC with grant No.10874151, 10935010, NFRPC with grant No. 2006CB921205; Program for New Century Excellent Talents in University (NCET), and Science Fundation of Chinese University.
[1] C. Liu, Z. Dutton, C. H. Behroozi, and L. V. Hau, Nature (London) 409, 490 (2001).

[2] M. D. Lukin, Rev. Mod. Phys. 75, 457 (2003).

[3] M. Fleischhauer and M. D. Lukin, Phys. Rev. A 65, 022314 (2002).

[4] M. Fleischhauer, S. F. Yelin, M. D. Lukin, Opt. Commun. 179, 395 (2000).

[5] C. P. Sun, Y. Li, and X. F. Liu, Phys. Rev. Lett. 91, 147903 (2003).

[6] R. H. Dicke, Phys. Rev. 93, 99 (1954).

[7] B. Zhao, Y. A. Chen, X. H. Bao, T. Strassel, C. S. Chuu, X. M. Jin, J. Schmiedmayer, Z. S. Yuan, S. Chen and J. W. Pan, Nature Phys. 5, 95 (2009).

[8] Y. O. Dudin, S. D. Jenkins, R. Zhao, D. N. Matsukevich, A. Kuzmich, and T. A. B. Kennedy, Phys. Rev. Lett. 103, 020505 (2009).

[9] M. D. Lukin, S. F. Yelin, and M. Fleischhauer, Phys. Rev. Lett. 84, 4232 (2000).

[10] W. H. Zurek, Rev. Mod. Phys. 75, 715 (2003).

[11] C. Mewes and M. Fleischhauer, Phys. Rev. A 72, 022327 (2005).

[12] J. Simon and H. Tanji, J. K. Thompson and V. Vuletić, Phys. Rev. Lett. 98, 183601 (2007).

[13] T. Yu and J. H. Eberly, Science 323, 598 (2009).

[14] X. Wang, A. Miranowicz, Y. X. Liu, C. P. Sun, and F. Nori, Phys. Rev. A 80, 022106 (2010).

[15] J. Appel, P. J. Windpassinger, D. Oblak, U. B. Hoff, N.
Kjægaard and E. S. Polzik, Proc. Nat. Acade. U. S. A. 106, 10960 (2009).

[16] M. Abramowitz and I. A. Stegun, Handbook of Mathematical Functions, (Dover, New York, 1965).

[17] M. Kitagawa and M. Ueda, Phys. Rev. A 47, 5138 (1993).

[18] D. J. Wineland, J. J. Bollinger, W. M. Itano, and D. J. Heinzen, Phys. Rev. A 50, 67 (1994).

[19] G. Tóth, C. Knapp, O. Gühne, and H. J. Briegel, Phys. Rev. Lett. 99, 250405 (2007); Phys. Rev. A 79, 042334 (2009).

[20] A. Sørensen, L. M. Duan, J. I. Cirac, and P. Zoller, Nature (London) 409, 63 (2001).

[21] X. Wang and B. C. Sanders, Phys. Rev. A 68, 012101 (2003).

[22] W. K. Wootters, Phys. Rev. Lett. 80, 2245 (1998).

[23] X. Wang and K. Mølmer, Eur. Phys. J. D 18, 385 (2002).

[24] C. Santori, D. Fattal, K. C. Fu, P. E. Barclay, and R. G. Beausoleil, New J. Phys. 11, 123009 (2009).

[25] S. S. Jang, Y. W. Cheong, J. Kim, and H. W. Lee, Phys. Rev. A 74, 062112 (2006).

[26] M. Weitz, B. C. Young and S. Chu, Phys. Rev. Lett. 73, 2563 (1994).

[27] R. G. Unanyan, B. W. Shore, and K. Bergmann, Phys. Rev. A 63, 043405 (2001).

[28] I. R. Solá, V. S. Malinovsky, and D. J. Tannor, Phys. Rev. A 60, 3081 (1999).

[29] C. Simon, M. Afzelius, J. Appel, A. Boyer de la Giro- 
day, S.J. Dewhurst, N. Gisin, C.Y. Hu, F. Jelezko, S. Kroll, J.H. Muller, J. Nunn, E. Polzik, J. Rarity, H. de Riedmatten, W. Rosenfeld, A.J. Shields, N. Skold, R.M. Stevenson, R. Thew, I. Walmsley, M. Weber, H. Weinfurter, J. Wrachtrup, and R.J. Young, arXiv:1003.1107.
[30] Shuai Chen, Yu-Ao Chen, Thorsten Strasse, Zhen-Sheng Yuan, Bo Zhao, Jörg Schmiedmayer, and Jian-Wei Pan, Phys. Rev. Lett. 97, 173004 (2006). 